\title{
Design of a hybrid model for cardiac arrhythmia classification based on Daubechies wavelet transform
}

\author{
Rekha Rajagopal ${ }^{B-F}$, Vidhyapriya Ranganathan ${ }^{A}$ \\ Department of Information Technology, PSG College of Technology, Coimbatore, India \\ A - research concept and design; $B$ - collection and/or assembly of data; $C$ - data analysis and interpretation; \\ $D$ - writing the article; $E$ - critical revision of the article; $F$ - final approval of the article
}

\author{
Address for correspondence \\ Rekha Rajagopal \\ E-mail: rekha.psgtech@gmail.com \\ Funding sources \\ None declared \\ Conflict of interest \\ None declared

\section{Acknowledgements} \\ The authors express their sincere and heartfelt \\ thanks to the management and to the principal \\ of PSG College of Technology, Coimbatore \\ for extending the essential support and \\ infrastructure to carry out this work.
}

Received on March 16, 2016 Reviewed on August 17, 2016 Accepted on February 14, 2017

\begin{abstract}
Background. Automation in cardiac arrhythmia classification helps medical professionals make accurate decisions about the patient's health.

Objectives. The aim of this work was to design a hybrid classification model to classify cardiac arrhythmias.

Material and methods. The design phase of the classification model comprises the following stages: preprocessing of the cardiac signal by eliminating detail coefficients that contain noise, feature extraction through Daubechies wavelet transform, and arrhythmia classification using a collaborative decision from the K nearest neighbor classifier (KNN) and a support vector machine (SVM). The proposed model is able to classify 5 arrhythmia classes as per the ANSI/AAMI EC57: 1998 classification standard. Level 1 of the proposed model involves classification using the KNN and the classifier is trained with examples from all classes. Level 2 involves classification using an SVM and is trained specifically to classify overlapped classes. The final classification of a test heartbeat pertaining to a particular class is done using the proposed KNN/SVM hybrid model.
\end{abstract}

Results. The experimental results demonstrated that the average sensitivity of the proposed model was $92.56 \%$, the average specificity $99.35 \%$, the average positive predictive value $98.13 \%$, the average $\mathrm{F}$-score $94.5 \%$, and the average accuracy $99.78 \%$.

Conclusions. The results obtained using the proposed model were compared with the results of discriminant, tree, and KNN classifiers. The proposed model is able to achieve a high classification accuracy.

Key words: support vector machine, biomedical data classification, decision support systems

DOI

10.17219/acem/68982

\section{Copyright}

Copyright by Author(s)

This is an article distributed under the terms of the

Creative Commons Attribution Non-Commercial License

(http://creativecommons.org/licenses/by-nc-nd/4.0/) 


\section{Introduction}

Cardiovascular disease is a leading cause of global mortality. Hence, there is a need to develop automation strategies for the management of sudden cardiac death. ${ }^{1}$ The objective of this work is to automate cardiac arrhythmia classification. An abnormality in the normal rhythm of a heartbeat causes arrhythmia. The ANSI/AAMI EC57: 1998 classification standard categorizes arrhythmias into 5 classes, namely: non-ectopic beat $(\mathrm{N})$, supra-ventricular ectopic beat (S), ventricular ectopic beat (V), fusion beat $(F)$, and unknown beat $(\mathrm{Q})$. The diagnosis of a specific class of arrhythmia is done by careful monitoring of a long-term electrocardiograph (ECG) signal. Automation in ECG arrhythmia classification is very essential in order to make a fast and accurate decision about the arrhythmia class. The key requirements of an automated system are reduced complexity, fast decision making, and less memory. Several research projects have been carried out for automation in arrhythmia classification. In general, the algorithm used for automated classification includes (i) preprocessing, (ii) feature extraction, and (iii) feature classification. The preprocessing of recorded ECG signals is done in order to eliminate the important noises that degrade the classifier performance, such as baseline wandering, motion artifact, power line interference, and high frequency noise. Currently, researchers use many filtering techniques like morphological filtering, integral coefficient band stop filtering, finite impulse response filtering, $5-20 \mathrm{~Hz}$ band pass filtering, median filtering, and wavelet-based denoising for preprocessing. ${ }^{2-7,9,25}$

Commonly extracted ECG features include (i) temporal features of heartbeat, such as the $\mathrm{P}-\mathrm{Q}$ interval, the QRS interval, the $\mathrm{S}-\mathrm{T}$ interval, the $\mathrm{Q}-\mathrm{R}$ interval, the $\mathrm{R}-\mathrm{S}$ interval, and the $\mathrm{R}-\mathrm{R}$ interval between adjacent heartbeats, (ii) amplitude-based features, such as $\mathrm{P}$ peak amplitude, $\mathrm{Q}$ peak amplitude, $\mathrm{R}$ peak amplitude, $\mathrm{S}$ peak amplitude, and $\mathrm{T}$ peak amplitude, (iii) wavelet transform-based features that include Haar wavelets, Daubechies wavelets, and discrete Meyer wavelets at various decomposition levels of 4, 6, and 8, and (iv) Stockwell transform-based features, including statistical features taken from a complex matrix of Stockwell transform, time-frequency contour and timemax amplitude contour.

A support vector machine (SVM), a probabilistic neural network (PNN), a multilayer perceptron neural network (MLPNN), a linear discriminant classifier, a mixture of experts, and unsupervised clustering are commonly used by researchers for the classification of ECG arrhythmia. ${ }^{5,6,9-16,21,24-26}$ Parameters such as accuracy, sensitivity, and specificity are used in the literature for evaluating the performance of a classifier. Most of the research works reported more than $90 \%$ average accuracy, average sensitivity, and average specificity taken over all 5 classes. However, the classifier outputs very poor sensitivity when the sensitivity of individual classes is considered. The reason is that, in a medical scenario, the number of training examples for each class of ECG arrhythmia may not be uniform. Usually, the normal class of heart beats dominates the entire population, which leads to biased classification towards classes with larger examples.

Some of the common limitations in the literature are listed as follows:

1. Time interval features are used in many automated systems. ${ }^{2,5,7,8}$ Hidden information in the ECG signal cannot be completely recovered from those time domain features.

2. Few researchers have used the entire data set of MIT_BIH arrhythmia database for experimentation. A random selection of only a few records from the entire database may not provide the actual result of their proposed system. ${ }^{2,5,7-9,23,24,28}$

3. A few research works did not follow a standard classification scheme, such as the ANSI/AAMI EC57: 1998 standard. $2,12,13,16,23,24$

4. Classes with major and minor training examples are treated equally in almost all projects, and this may lead to biased results towards major classes. The distinction between ventricular ectopic beats and supra-ventricular ectopic beats should be considered very important because some drugs for supraventricular ectopic beats can worsen the clinical state if the rhythm is a ventricular ectopic beat.

5. Class $\mathrm{N}$ and class $\mathrm{S}$ show a highly overlapped pattern. No special care is taken to overcome this issue.

This work eliminates the above limitations by extracting features from the time-frequency representation of an ECG signal through wavelet transform. The entire dataset of a benchmark database (i.e., the MIT_BIH arrhythmia database) is used and the proposed model adheres to the classification standard. The proposed model trains the classifier in such a way that the classifier better predicts the minority class using a hybrid approach.

\section{Material and methods}

The MIT_BIH arrhythmia database was used in this work. It contains 48 half-h excerpts of 2-channel ambulatory ECG recordings which were obtained from 47 subjects studied by the BIH arrhythmia laboratory. The recordings were digitized at 360 samples per second per channel with 11-bit resolution over a $10-\mathrm{mV}$ range. The reference annotations for each beat were included in the database. Four records containing paced beats $(102,104,107$, and 217) were removed from the analysis as specified by the AAMI. The total number of heart beats in each class is given in Table 1. Figure 1 shows the architecture of the proposed work. The entire experiments were carried out using Matlab R2012a (MathWorks, Natick, USA). The details of the methodology followed are summarized below. 
Table 1. Number of heartbeats in each class

\begin{tabular}{|l|c|c|c|c|c|}
\hline Heartbeat type & N & S & V & F & Q \\
\hline Full database & 87643 & 2646 & 6792 & 794 & 15 \\
\hline
\end{tabular}

$\mathrm{N}$ - non-ectopic beat; S - supra-ventricular ectopic beat; $\mathrm{V}$ - ventricular ectopic beat; F - fusion beat; Q - unknown beat.

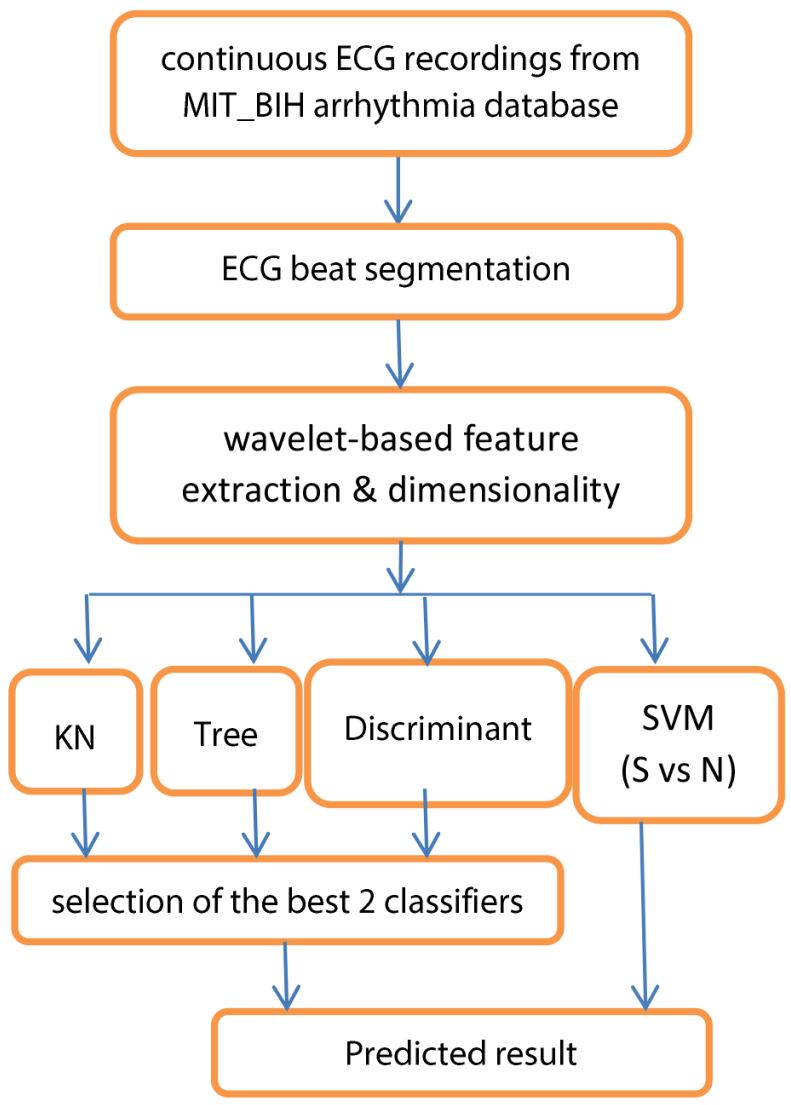

Fig. 1. Architecture of the proposed work

\section{Data preprocessing}

The records contain continuous ECG recordings of a duration of $30 \mathrm{~min}$. The raw ECG signals include baseline wander, motion artifact, and power line interference noise. The discrete wavelet transform (DWT) is used for denoising the ECG signal and for extracting the important features from the original ECG signal. ${ }^{22,27}$ The DWT captures both temporal and frequency information. The DWT of the original ECG signal is computed by successive high pass and low pass filtering of that signal. This can be mathematically represented as follows in equations (1) and (2),

(1) $\mathrm{y}_{\text {high }}[\mathrm{k}]=\sum_{\mathrm{n}}^{\infty}={ }_{-\infty} \mathrm{x}[\mathrm{n}] \mathrm{g}[2 \mathrm{k}-\mathrm{n}]$

(2) $\mathrm{y}_{\text {low }}[\mathrm{k}]=\sum_{\mathrm{n}}^{\infty}={ }_{-\infty} \mathrm{x}[\mathrm{n}] \mathrm{h}[2 \mathrm{k}-\mathrm{n}]$

where $x[n]$ is the original ECG signal samples, $g$ and $h$ are the impulse responses of the high pass and low pass filters, respectively, and are the outputs of the high pass and low pass filters after sub-sampling by 2 . This procedure is repeated until the required decomposition level is reached. The low frequency component is called approximation and the high frequency component is called detail.

In this work, the raw ECG signals sampled at $360 \mathrm{~Hz}$ were decomposed into approximation and detail sub bands up to level 9 using Daubechies ('db8') wavelet basis function. ${ }^{18}$ The first and second level detail coefficients were made zero and were not used for reconstruction of the denoised signal, since most of the ECG information is contained within the $40-\mathrm{Hz}$ frequency range and sub bands at the first and second levels contain the frequency ranges $90-180 \mathrm{~Hz}$ and $45-90 \mathrm{~Hz}$, respectively. Moreover, power line interference noise occurs at $50 \mathrm{~Hz}$ or $60 \mathrm{~Hz}$. Baseline wander noise occurs in the frequency range of $<0.5 \mathrm{~Hz}$, and therefore, the level 9 approximation sub band in the frequency range of $0-0.351 \mathrm{~Hz}$ was not used for reconstruction. The denoised signal was obtained by applying inverse DWT to the required detail coefficients of levels $3,4,5,6,7,8$, and 9 . The coefficients of detail sub bands 1 and 2 and the approximation sub band 9 were made 0 .

After denoising, the continuous ECG waveform was segmented into individual heartbeats. This segmentation is done by identifying the $\mathrm{R}$ peaks using the Pan-Tompkins algorithm and by considering the 99 samples before the $\mathrm{R}$ peak and the 100 samples after the R peak. ${ }^{19}$ This choice of 200 samples, including the $\mathrm{R}$ peak for segmentation, was made because it constitutes one cardiac activity with $\mathrm{P}, \mathrm{QRS}$, and T waves. Figure 2 shows a segment of a recorded ECG waveform of patient No. 123 before and after preprocessing.

\section{Feature extraction}

The entire database $(97,890$ heartbeats) is divided into 10 sets, each containing 9,789 heartbeats. Nine sets are used for training $(88,101$ heartbeats) and 1 set for testing (9,789 heartbeats). From each heartbeat, wavelet-based features are extracted by using Daubechies wavelet ('db4'). A Daubechies wavelet with level 4 decomposition was selected in this project after making performance comparisons with a discrete Meyer wavelet and other levels of Daubechies wavelets, including 'db2' and 'db6'. A total of 107 features were produced by the $4^{\text {th }}$ level approximation sub-band and another 107 features by the $4^{\text {th }}$ level detail sub-band. Principal component analysis (PCA) was applied to reduce redundant information on the extracted features and to reduce the dimensionality. After dimensionality reduction was applied separately to the approximation and detail sub-bands, a total of 12 features were obtained. The choice of 6 features from each sub-band was made since there is no significant improvement in classification when more than 6 features are used. 

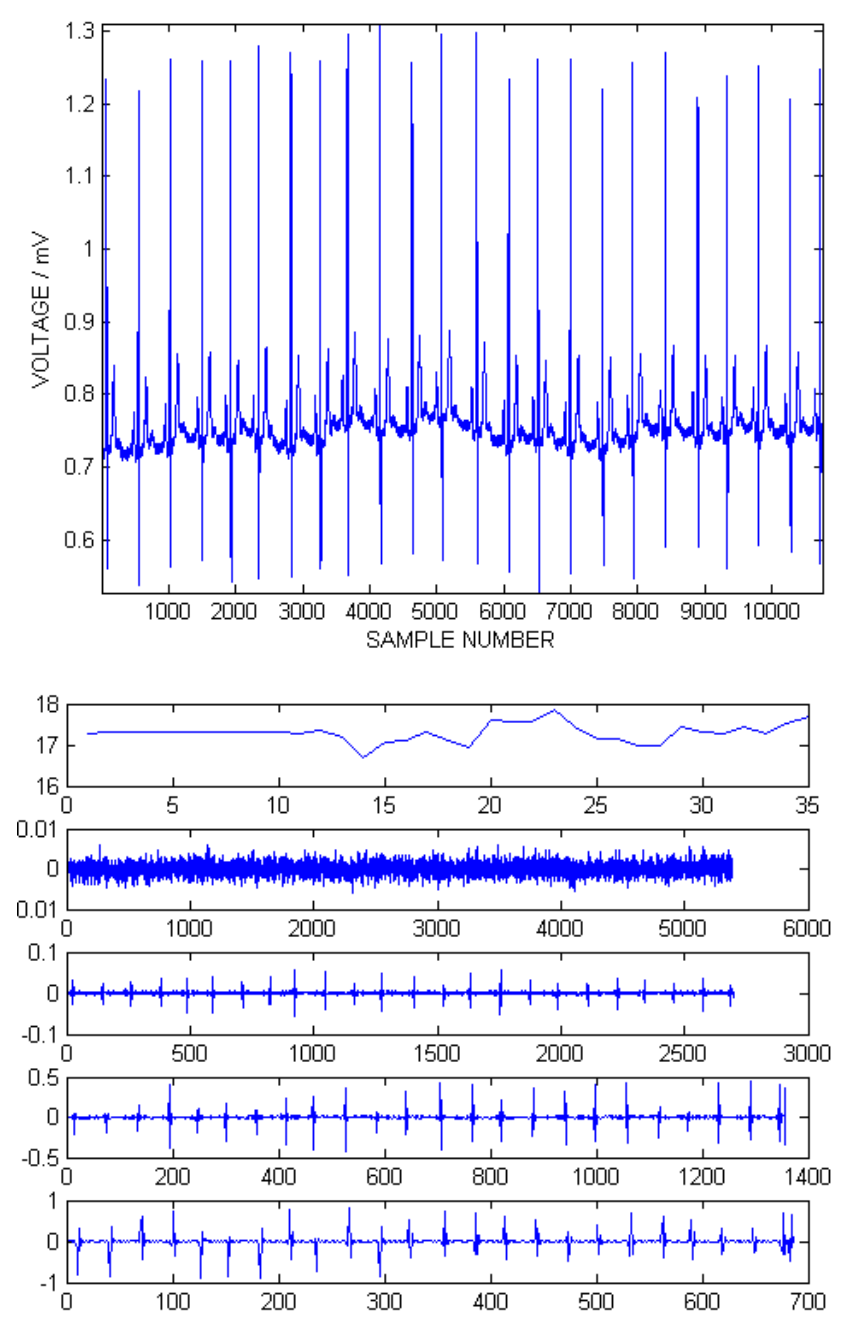

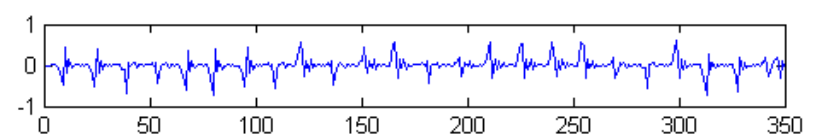

\begin{tabular}{llllllll}
\hline & 50 & 100 & 150 & 200 & 250 & 300 & 350
\end{tabular}

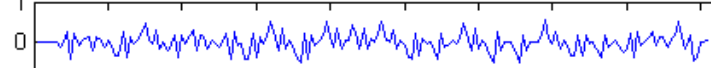

$\begin{array}{rrrrrrrrrrr}-1 & & 1 & 1 & 1 & 1 & 1 & 1 & 1 & 1 & 1 \\ 10 & 20 & 40 & 60 & 80 & 100 & 120 & 140 & 160 & 180 & 200\end{array}$

${ }_{-0.5}^{0.5} \wedge N_{1}$

(10)
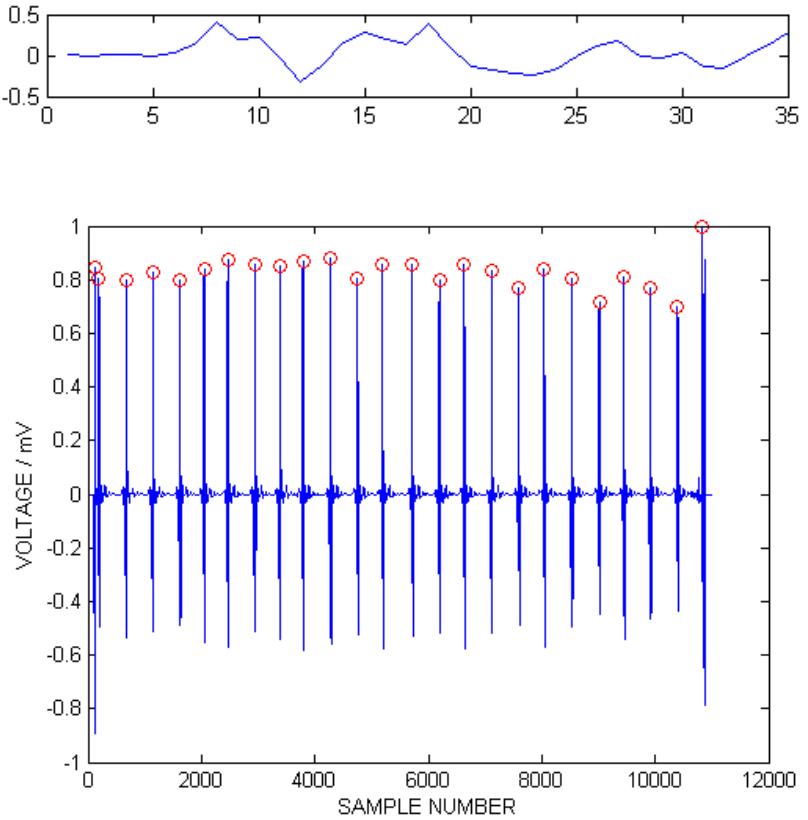

Fig. 2. A segment of an ECG waveform before and after preprocessing

A - raw ECG signal; B - approximation subband level 9 and detail subband levels 1-4 (bottom left corner); C - detail subband levels 5-9 (top right corner); $\mathrm{D}$ - preprocessed ECG waveforms with R peaks detected.

\section{Training of classifiers}

The training and testing matrix was computed, in which each row represents an ECG heartbeat and the features occupy the columns. The KNN (with distance metrics such as Euclidean, correlation, Mahalanobis, standardized Euclidean, and Spearman), tree, and discriminant (linear and quadratic) classifiers are trained with a training matrix $88,101 \times$ 12 in size, which includes training examples from all 5 classes. The sensitivity, specificity, accuracy, positive predictivity, and F-score of those classifiers in classifying ECG arrhythmias were compared. The classifier that produced the best sensitivity and F-score was selected at level 1 of the proposed model. The radial basis function SVM was used at level 2 of the proposed model and was trained with examples from the entire class $\mathrm{S}$ and down-sampled examples from class N. Random down-sampling of class $\mathrm{N}$ is done in order to match the sample size of class $S(2,646 \times 12)$. The reason for this design is that samples from classes $\mathrm{S}$ and $\mathrm{N}$ are highly overlapped and many class $\mathrm{S}$ samples are wrongly predicted as class $\mathrm{N}$ at level 1 because of the large number of class $\mathrm{N}$ training examples $(87,643 \times 12)$. More weight is given to a decision from the SVM classifier while determining a test heartbeat to be other than class S. The advantage of the SVM classifier is that it performs well on datasets that have many attributes, even when there are few training examples available. But the drawback of SVM is its limitation in speed and size during both training and testing. Because of this limitation, SVM is not used for the training and classification of all classes. SVM is used only to make a final decision of a highly overlapped minority class. A description of the classifiers used is discussed in the following sections.

\section{K nearest neighbor classifier}

KNN is an instance-based simple classification algorithm. For a training data set of $\mathrm{N}$ points and its corresponding labels, given by $\left\{\left(\mathrm{x}_{1}, \mathrm{y}_{1}\right),\left(\mathrm{x}_{2}, \mathrm{y}_{2}\right) \ldots\left(\mathrm{x}_{\mathrm{N}}, \mathrm{y}_{\mathrm{N}}\right)\right\}$, 
where $\left(x_{i}, y_{i}\right)$ represents a data pair ' $i$ ' with ' $x_{i}$ ' as the input feature vector and ' $y_{i}^{\prime}$ ' as its corresponding target class label, the most likely class of test beat ' $x$ ' is determined by finding the $\mathrm{K}$ closest training points to it. The prediction of a class is determined by majority vote. The distance is taken as the weighting factor for voting. The main advantage in selecting the KNN classifier is that complex tasks can be learned using simple procedures by local approximation. The training process for KNN only consists of storing feature vectors and their corresponding labels. It also works well on classes with different characteristics for different subsets. ${ }^{20}$

\section{Tree-based classifier}

The decision tree algorithm works by selecting the best attribute to split the data and expand the leaf nodes of the tree until the stopping criterion is met. After building the tree, tree pruning is performed to reduce the size of the decision tree. This is done in order to avoid overfitting and to improve the generalization capability of decision trees. The class of a test heartbeat is determined by following the branches of the tree until a leaf node is reached. The class of that leaf node is then assigned to the test heartbeat. The advantage of this algorithm is its simplicity and good performance for larger data sets. Gini's diversity index is used as the split criterion in this work.

\section{Discriminant classifier}

The algorithm creates a new variable from one or more linear combinations of input variables. Linear discriminant analysis is done by calculating the sample mean of each class. Sample covariance is calculated by subtracting the sample mean of each class from the observations of that class, and taking the empirical covariance matrix of the result. In the linear discriminant model, only the means vary for each class, but the covariance matrix remains the same. For quadratic discriminant analysis, both the mean and covariance of each class varies.

\section{Support vector machine}

The support vector machine (SVM) constructs a hyper plane in such a way that the margin of separation between positive examples (minority class $\mathrm{S}$ ) and negative examples (majority class N) is maximized. Since classes $\mathrm{S}$ and $\mathrm{N}$ overlap very much, the hyper plane cannot be linearly separable and cannot be constructed without a classification error. For such overlapped patterns, SVM performs nonlinear mapping of the input vector into a high dimensional feature space. An optimal hyper plane is constructed for separation of these newly mapped features. The hyper plane is constructed in such a way that it minimizes the probability of a classification error. For a training set $\mathrm{X}$ with $\mathrm{N}$ number of training examples, if $\left\{\left(\mathrm{x}_{\mathrm{i}}, \mathrm{d}_{\mathrm{i}}\right)\right\}$ is the $\mathrm{i}^{\text {th }}$ training example, where $x_{i}$ is the input vector for the $i^{\text {th }}$ example and $d_{i}$ is its corresponding target output, $\alpha_{i}$ is the $i^{i \text { th }}$ Lagrange multiplier, $\mathrm{K}\left(\mathrm{x}, \mathrm{x}_{\mathrm{i}}\right)$ is the inner product kernel, and $b$ is the bias, then the optimal separating hyper plane is defined as in Equation (3):

(3) $\mathrm{f}(\mathrm{x})=\sum_{i=1}^{N} a_{1} d_{1} K\left(x, x_{i}\right)+b$

A radial basis function SVM was used in this work instead of polynomial and two-layer perceptron because of its higher discrimination ability. The inner product kernel $\mathrm{K}\left(\mathrm{x}, \mathrm{x}_{\mathrm{i}}\right)$ of a radial basis function with width $\sigma$ is given by equation (4):

(4) $K\left(x, x_{i}\right)=\exp \left(\frac{-1}{2^{\wedge 2}}\left\|x-x_{i}\right\|^{2}\right)$

The performance of the proposed model was evaluated using performance metrics such as sensitivity, specificity, positive predictivity, F-score, and accuracy. These metrics are computed by calculating true positive (TP), true negative (TN), false positive (FP), and false negative (FN) counts and are defined as follows: sensitivity $=\mathrm{TP} /(\mathrm{TP}$ $+\mathrm{FN})$, specificity $=\mathrm{TN} /(\mathrm{TN}+\mathrm{FP})$, positive predictivity $=\mathrm{TP} /(\mathrm{TP}+\mathrm{FP}), \mathrm{F}$-score $=2 \mathrm{TP} /(2 \mathrm{TP}+\mathrm{FP}+\mathrm{FN})$, and accuracy $=(\mathrm{TP}+\mathrm{TN}) /(\mathrm{TP}+\mathrm{FP}+\mathrm{FN}+\mathrm{TN})$. The process is repeated 10 times so that each set is used once for testing. The overall performance of the classifier is computed by taking the average of all 10 folds.

\section{Results and discussion}

The reliability of a classifier in accurately predicting the test heartbeat's class is measured mainly by the sensitivity and F-score. The reason for not considering accuracy is that even a poor classifier can show good accuracy in favoring a class with more training examples. It can be observed from Fig. 3 that a discriminant classifier with linear and quadratic function produces consistently less sensitivity than KNN and tree classifiers. The KNN with Euclidean distance metric produces the highest sensitivity.

Figure 4 shows the specificity of all classifiers in each of the 10 folds. The discriminant classifier produces the least specificity. The KNN classifier produces the highest specificity. Figure 5 shows the F-score of all classifiers in all 10 folds. The discriminant classifier with a linear function produces the lowest F-score. The tree classifier and the quadratic discriminant classifier produce a nearly uniform F-score, while the KNN classifier achieves the highest F-score.

The KNN with Euclidean distance metric achieves the highest accuracy compared to other classifiers and is shown in Fig. 6.

Table 2 shows the average classification results of all classifiers at level 1 . One can see from Table 2 that the KNN with Euclidean distance metric and 4 neighbors produces 


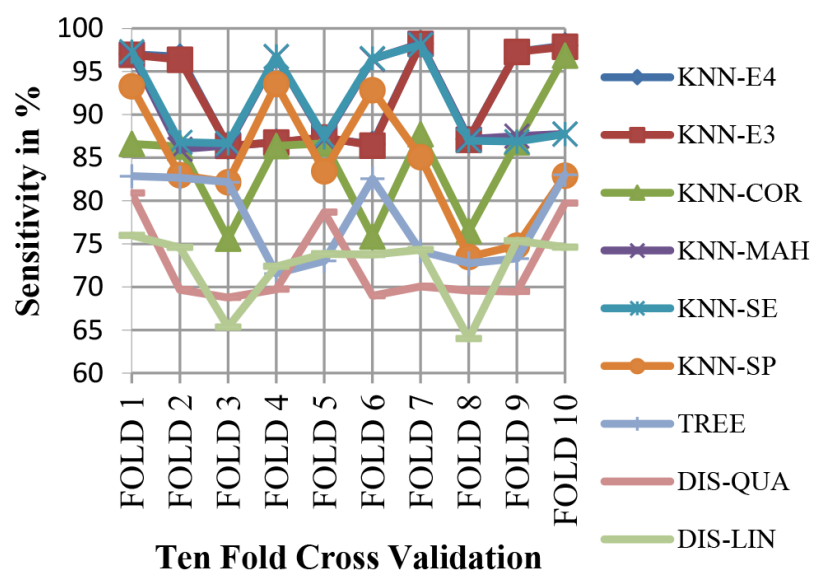

Fig. 3. Results of sensitivity for 10 different folds of KNN, tree, and discriminant classifiers

KNN-E4 - K-nearest neighbour classifier with Euclidean distance 4; KNN-E3 - K-nearest neighbour classifier with Euclidean distance 3; KNN-COR - K-nearest neighbour classifier with correlation distance metric KNN-MAH - K-nearest neighbour classifier with Mahalanobis distance metric; KNN-SE - K-nearest neighbour classifier with standardized Euclidean distance metric; KNN-SP - K-nearest neighbour classifier with Spearman distance metric; TREE - tree classifier; DIS-QUA - discriminant classifier with quadratic function; DIS-LIN - discriminant classifier with linear function.

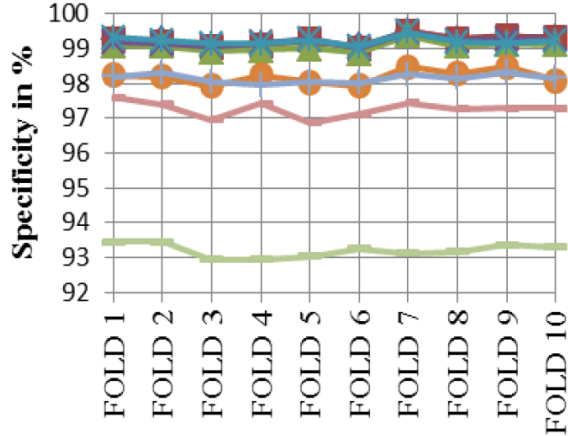

Ten Fold Cross Validation

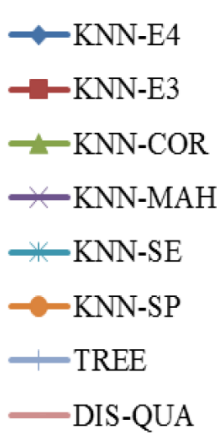

DIS-LIN
Fig. 4. Results of specificity for 10 different folds of KNN, tree, and discriminant classifiers

KNN-E4 - K-nearest neighbour classifier with Euclidean distance 4; KNN-E3 - K-nearest neighbour classifier with Euclidean distance 3; KNN-COR - K-nearest neighbour classifier with correlation distance metric; KNN-MAH - K-nearest neighbour classifier with Mahalanobis distance metric; KNN-SE - K-nearest neighbour classifier with standardized Euclidean distance metric; KNN-SP - K-nearest neighbour classifier with Spearman distance metric; TREE - tree classifier; DIS-QUA - discriminant classifier with quadratic function; DIS-LIN - discriminant classifier with linear function.

a better sensitivity, specificity, positive predictivity, F-score, and accuracy than the other 2 classifiers which were considered. Hence, $\mathrm{KNN}$ is used at level 1 of the proposed model. KNN with 3 neighbors also produces comparable results to $\mathrm{KNN}$ with 4 neighbors. Compared to $\mathrm{KNN}$ with 4 neighbors, the KNN classifier with 3 neighbors has a greater discrimination capability for class $\mathrm{S}$.

From the confusion matrix obtained from tenfold cross validation using different classifiers, it was found that a high number of class $\mathrm{S}$ heartbeats are misclassified

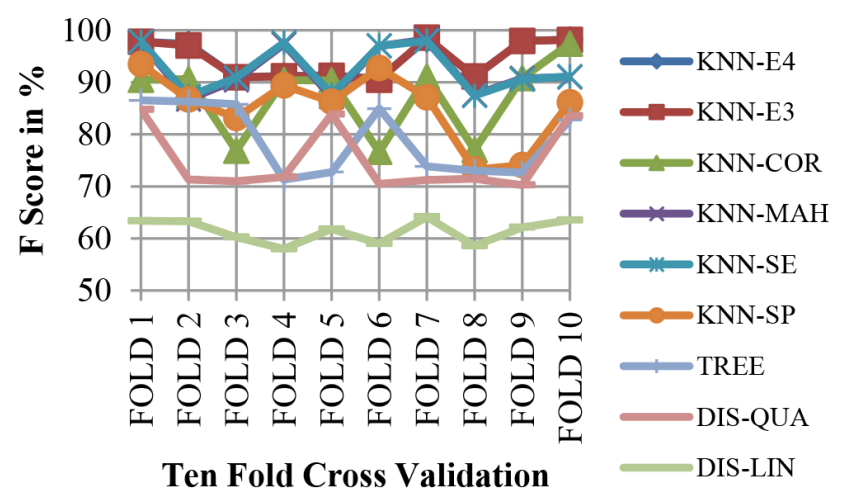

Fig. 5. Results of F-score for 10 different folds of KNN, tree, and discriminant classifiers

KNN-E4 - K-nearest neighbour classifier with Euclidean distance 4; KNN-E3 - K-nearest neighbour classifier with Euclidean distance 3; KNN-COR - K-nearest neighbour classifier with correlation distance metric; KNN-MAH - K-nearest neighbour classifier with Mahalanobis distance metric; KNN-SE - K-nearest neighbour classifier with standardized Euclidean distance metric; KNN-SP - K-nearest neighbour classifier with Spearman distance metric; TREE - tree classifier; DIS-QUA - discriminant classifier with quadratic function; DIS-LIN - discriminant classifier with linear function.

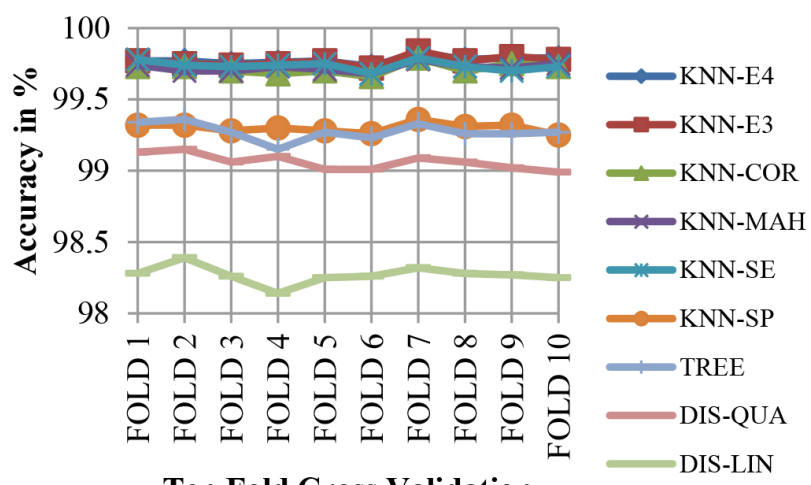

Ten Fold Cross Validation

Fig. 6. Results of accuracy for 10 different folds of KNN, tree, and discriminant classifiers

KNN-E4 - K-nearest neighbour classifier with Euclidean distance 4; KNN-E3 - K-nearest neighbour classifier with Euclidean distance 3; KNN-COR - K-nearest neighbour classifier with correlation distance metric; KNN-MAH - K-nearest neighbour classifier with Mahalanobis distance metric; KNN-SE - K-nearest neighbour classifier with standardized Euclidean distance metric; KNN-SP - K-nearest neighbour classifier with Spearman distance metric; TREE - tree classifier; DIS-QUA - discriminant classifier with quadratic function; DIS-LIN - discriminant classifier with linear function.

as normal class $\mathrm{N}$. This is because of the close resemblance of class $S$ to the normal class and because the number of training examples for class $\mathrm{N}$ is higher than class $\mathrm{S}$. A sample confusion matrix of the KNN classifier with 4 neighbors at level 1 is shown in Table 3.

Moreover, the number of training examples for class $\mathrm{N}(78,879$ heartbeats) is much greater than class $\mathrm{S}(2,381$ heartbeats) training examples. This may be the reason why the KNN with 3 neighbors can classify class $\mathrm{S}$ better than the KNN with 4 neighbors. For other classes, KNN with 4 neighbors performed well. To achieve better results, the SVM classifier is used along with KNN. SVM is trained 
Table 2. Average classification results of tenfold cross validation for classifiers at level 1

\begin{tabular}{|c|c|c|c|c|c|}
\hline Classifier & 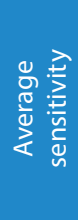 & 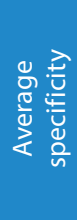 & 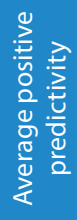 & 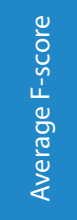 & 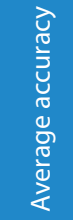 \\
\hline KNN-Euclidean 4 & 92.14 & 99.24 & 98.56 & 94.48 & 99.77 \\
\hline KNN-Euclidean 3 & 92.07 & 99.23 & 98.53 & 94.43 & 99.76 \\
\hline KNN-correlation & 84.53 & 99.07 & 97.92 & 87.16 & 99.71 \\
\hline KNN-Mahalanobis & 91.05 & 99.21 & 94.74 & 92.39 & 99.72 \\
\hline KNN-standardized Euclidean & 91.11 & 99.22 & 94.97 & 92.53 & 99.73 \\
\hline KNN-Spearman & 84.45 & 98.19 & 89.09 & 85.23 & 99.30 \\
\hline Tree classifier & 77.80 & 98.15 & 83.72 & 78.98 & 99.28 \\
\hline Discriminant-linear & 72.42 & 93.20 & 60.87 & 61.41 & 98.28 \\
\hline Discriminant-quadratic & 72.56 & 97.27 & 92.68 & 74.97 & 99.06 \\
\hline
\end{tabular}

Table 5. Sensitivity and F-score of class S before and after using the proposed model

\begin{tabular}{|l|c|c|c|c|}
\hline \multirow{2}{*}{$\begin{array}{l}\text { Fold } \\
\text { number }\end{array}$} & \multicolumn{2}{|c|}{ Sensitivity of class S } & \multicolumn{2}{c|}{ F-Score of class S } \\
\cline { 2 - 5 } & KNN - EUC 4 & Proposed & KNN - EUC 4 & Proposed \\
\hline Fold 1 & 88.68 & 90.56 & 92.16 & 92.30 \\
\hline Fold 2 & 88.30 & 91.32 & 91.40 & 93.07 \\
\hline Fold 3 & 85.28 & 87.54 & 90.58 & 90.62 \\
\hline Fold 4 & 88.67 & 91.69 & 92.33 & 92.74 \\
\hline Fold 5 & 88.67 & 90.56 & 91.79 & 92.13 \\
\hline Fold 6 & 85.66 & 88.30 & 89.90 & 90.00 \\
\hline Fold 7 & 92.07 & 93.58 & 94.02 & 93.23 \\
\hline Fold 8 & 89.43 & 90.56 & 92.75 & 92.13 \\
\hline Fold 9 & 90.18 & 91.69 & 93.35 & 92.57 \\
\hline Fold 10 & 92.07 & 94.33 & 93.84 & 93.80 \\
\hline
\end{tabular}

KNN-EUC 4 - K-nearest neighbour classifier with Euclidean distance 4.
Table 3. Confusion matrix (KNN 4 fold 2)

\begin{tabular}{c|c|c|c|c|c|c|}
$\begin{array}{c}n=9789 \text { (where } \\
\text { ' } n \text { ' is the total } \\
\text { number of test } \\
\text { samples in fold 2) }\end{array}$ & \multicolumn{5}{|c|}{ Predicted } \\
\hline & arrhythmia class & S & V & F & N & Q \\
\hline S & 234 & 1 & 0 & 30 & 0 \\
\hline Actual & V & 0 & 674 & 2 & 3 & 0 \\
\hline F & 0 & 0 & 76 & 3 & 0 \\
\hline N & 13 & 2 & 1 & 8748 & 0 \\
\hline Q & 0 & 0 & 0 & 0 & 2 \\
\hline
\end{tabular}

$\mathrm{N}$ - non-ectopic beat; $\mathrm{S}$ - supra-ventricular ectopic beat; $\mathrm{V}$ - ventricular ectopic beat; $\mathrm{F}$ - fusion beat; Q - unknown beat.

Table 4. Confusion matrix (proposed model fold 2)

\begin{tabular}{c|c|c|c|c|c|c|}
$\begin{array}{c}n=9789 \text { (where } \\
\text { ' } \mathrm{n} \text { ' is the total } \\
\text { number of test } \\
\text { samples in fold 2) }\end{array}$ & \multicolumn{5}{|c|}{ Predicted } \\
\hline & arrhythmia class & S & V & F & N & Q \\
\hline S & 242 & 1 & 0 & 22 & 0 \\
\hline Actual & V & 0 & 674 & 2 & 3 & 0 \\
\hline F & 0 & 0 & 76 & 3 & 0 \\
\hline N & 13 & 2 & 1 & 8748 & 0 \\
\hline
\end{tabular}

$\mathrm{N}$ - non-ectopic beat; $\mathrm{S}$ - supra-ventricular ectopic beat; $\mathrm{V}$ - ventricular ectopic beat; $\mathrm{F}$ - fusion beat; Q - unknown beat.

to classify class $\mathrm{S}$ from class $\mathrm{N}$. The classification result of KNN with Euclidean distance metric (4 and 3 neighbors) was compared with the predicted result of Support vector machine (SVM). A test heartbeat is concluded to be class $S$ if at least 2 classifiers predict it as class $\mathrm{S}$. A sample confusion matrix of the proposed hybrid model is shown in Table 4.
Sensitivity and F-score results before and after the usage of the proposed model are shown in Table 5. It is clear that the sensitivity of class $\mathrm{S}$ improves considerably through this hybrid model. The performance of other classes remains unaltered and the classification performance of class $\mathrm{N}$ gets slightly reduced by an average sensitivity percentage of 0.09 and an average F-score percentage of 0.01 . Class $\mathrm{N}$ is the normal class and the problem of normal class misclassified as class $\mathrm{S}$ is less compared to a class $S$ beat misclassified as normal class N. Hence, the proposed model improves sensitivity in the prediction of all heartbeat classes.

\section{Conclusions}

In this paper, a hybrid classification model is proposed which inherits the abilities of both SVM and KNN. Instead of using a simple classifier as KNN for predicting highly overlapped classes, this mixed model improves the sensitivity of minority classes, which is dominated by the majority class. SVM is specifically trained to classify overlapped classes. At the same time, the low complex KNN classifier is trained to classify all 5 classes. Hence, the final decision of a test heartbeat is done using classifiers at both levels of the hierarchy. The performance of this model is supported by experimental results on the entire MIT/BIH arrhythmia database. Future work will experiment with other combinations of classifiers. 


\section{References}

1. Mehra R. Global public health problem of sudden cardiac death. J Electrocardiol. 2007; 40(6 Suppl):118-122.

2. Kim J, Shin HS, K, Shin, Lee M. Robust algorithm for arrhythmia classification in ECG using extreme learning machine. Bio Medical Engineering Online. 2009. https://doi.org/10.1186/1475-925X-8-31

3. Liang W, Zhang Y, Tan J, Li Y. A novel approach to ECG classification based upon two-layered HMMs in body sensor networks. Sensors. 2014;14(4):5994-6011.

4. Kim H, Yazicioglu RF, Merken P, Van Hoof C, Yoo HJ. ECG signal compression and classification algorithm with quad level vector for ECG holter system. IEEE Trans Inf Technol Biomed. 2010;14(1):93-100.

5. Asl BM, Setarehdan SK, Mohebbi M. Support vector machine - based arrhythmia classification using reduced features of heart rate variability signal. Artif Intell Med. 2008;1(44):51-64.

6. Huang K, Zhang L. Cardiology knowledge free ECG feature extraction using generalized tensor rank one discriminant analysis. EURASIP J App/Signal Processing. 2014. https://doi.org/10.1186/1687-6180-2014-2

7. Zhu B, Ding Y, Hao K. A novel automatic detection for ECG arrhythmias using maximum margin clustering with immune evolutionary algorithm. Comput Math Methods Med. 2013. http://dx.doi. org/10.1155/2013/453402

8. Chazal P, Dwyer MO, Reilly RB. Automatic classification of heartbeats using ECG morphology and heartbeat interval features. IEEE Trans Biomed Eng. 2004;7(51):1196-1206.

9. Roshan Joy Martis, Rajendra Acharya U, Lim Choo Min. ECG beat classification using PCA, LDA, ICA and discrete wavelet transform. Biomed Signal Process Control. 2013;5(8):437-448.

10. Manab Kumar Das, Samit Ari. ECG beats classification using mixture of features. Int Sch Res Notices. 2014;2014:178436. doi: $10.1155 / 2014 / 178436$

11. Das MK, Ari S. Electrocardiogram beat classification using S-Transform based feature set. J Mech Med Biol. 2014;5(14):1450066.

12. Rai HM, Trivedi A, Shukla S. ECG signal processing for abnormalities detection using multi-resolution wavelet transform and artificial neural network classifier. Measurement. 2013;9(46):3238-3246.

13. Song MH, Lee J, Lee KJ, Yoo SK. Support vector machine based arrhythmia classification using reduced features. Int J Control Autom. 2005;4(3):571-579.

14. Zidelmal Z, Amirou A, Ould Abdeslam D, Merckle J. ECG beat classification using a cost sensitive classifier. Comput Meth Prog Bio. 2013;3(111):570-577.

15. Daamouche A, Hamami L, Alajlan N, Melgani F. A wavelet optimization approach for ECG signal classification. Biomed Signal Process Control. 2012;(7):342 -349.

16. Ubeyli ED. Usage of Eigen vector methods in implementation of automated diagnostic systems for ECG beats. Digit Signal Process. 2008;33(18):33-48.

17. Moody GB, Mark RG. The impact of the MIT_BIH arrhythmia database. IEEE Eng Med Biol. 2001;3(20):45-50.

18. Singh BN, Tiwari AK. Optimal selection of wavelet basis function applied to ECG signal denoising. Digit Signal Process. 2006;3(16): 275-287.

19. Pan J, Tompkins JW. A real time QRS detection algorithm. IEEE Trans Biomed Eng. 1985;3(32). https://doi.org/10.1109/TBME.1985.325532

20. Kim J, Kim BS, Savarese S. Comparing image classification methods: K Nearest Neighbor and Support Vector Machines. Applied Mathematics in Electrical and Computer Engineering. 2012; 133-138.

21. Ghoraani B, Krishnan S. Discriminant non-stationary signal features clustering using hard and fuzzy cluster labeling. EURASIP J Adv Signal Process. 2012. https://doi.org/10.1186/1687-6180-2012-250

22. Mazomenos EB, Biswas D, Acharyya A, et al. A low-complexity ECG feature extraction algorithm for mobile healthcare applications. IEEE J Biomed Health Inform. 2013;2(17):459-569.

23. Sufi F, Khalil I,Mahmood AN. A clustering based system for instant detection of cardiac abnormalities from compressed ECG. Expert Syst Appl. 2011;5(38):4705-4713.

24. Melgani F, Bazi Y. Classification of electrocardiogram signals with support vector machines and particle swarm optimization. IEEE Trans Inf Technol Biomed. 2008;5(12):667-677.
25. Oresko JJ, Jin Z, Huang S, Sun Y, Duschl, Cheng AC. A wearable smart phone based platform for real time cardiovascular disease detection via electrocardiogram processing. IEEE Trans Inf Technol Biomed. 2010;3(14):734-740.

26. Wiens J, Guttag JV. Patient adaptive ectopic beat classification using active learning. Comput Cardiol. 2010;37:109-112.

27. Li D, Pedrycz W, Pizzi JN. Fuzzy wavelet packet based feature extraction method and its application to biomedical signal classification. IEEE Trans Biomed Eng. 2005;6(52):1132-1139.

28. Luz E, Menotti D. How the choice of samples for building arrhythmia classifiers impact their performances. Proc Conf IEEE Eng Med Biol Soc. 2011;4988-4991. 